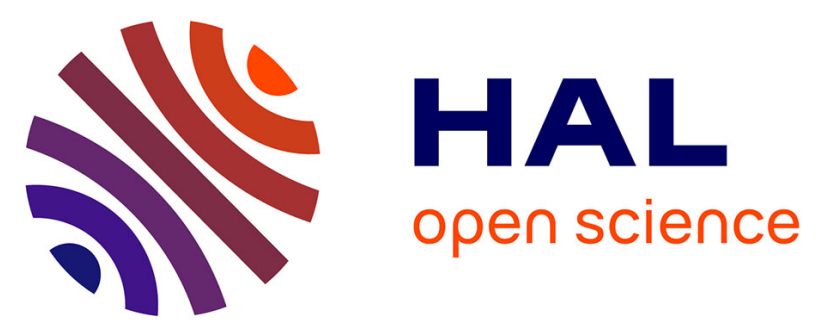

\title{
POTENTIAL DISTRIBUTION IN METAL-VACUUM-METAL PLANAR BARRIERS CONTAINING SPHERICAL PROTRUSIONS OR INCLUSIONS
}

A. Lucas, J. Vigneron, J. Bono, P. Cutler, T. Feuchtwang, R. Good, Jr. Huang, Z. Huang

\section{To cite this version:}

A. Lucas, J. Vigneron, J. Bono, P. Cutler, T. Feuchtwang, et al.. POTENTIAL DISTRIBUTION IN METAL-VACUUM-METAL PLANAR BARRIERS CONTAINING SPHERICAL PROTRUSIONS OR INCLUSIONS. Journal de Physique Colloques, 1984, 45 (C9), pp.C9-125-C9-132. 10.1051/jphyscol:1984922 . jpa-00224401

\section{HAL Id: jpa-00224401 https://hal.science/jpa-00224401}

Submitted on 1 Jan 1984

HAL is a multi-disciplinary open access archive for the deposit and dissemination of scientific research documents, whether they are published or not. The documents may come from teaching and research institutions in France or abroad, or from public or private research centers.
L'archive ouverte pluridisciplinaire HAL, est destinée au dépôt et à la diffusion de documents scientifiques de niveau recherche, publiés ou non, émanant des établissements d'enseignement et de recherche français ou étrangers, des laboratoires publics ou privés. 


\title{
POTENTIAL DISTRIBUTION IN METAL-VACUUM-METAL PLANAR BARRIERS CONTAINING SPHERICAL PROTRUSIONS OR INCLUSIONS +
}

\author{
A.A. Lucas, J.P. Vigneron, J. Bono ${ }^{\star}$, P.H. Cutler ${ }^{\star}$, T.E. Feuchtwang $\star$, \\ R.H. Good, Jr* and Z. Huang ${ }^{\star}$ \\ Départment de Physique, F.N.D.P., 61, rue de Bruxezzes, 5000 Namur, \\ Belgium \\ * Physics Department, The Pennsylvania State University, University Park, \\ Pennsy Uuania 16802, U.S.A.
}

\begin{abstract}
Résumé - On prêsente une méthode exacte pour le calcul rapide a) de 1 a distribution de potentiel ëlectrostatique rëgnant entre deux électrodes planes parallèles contenant une protrusion sphérique ou insertion sphérique conductrice et soumises à une différence de potentiel et b) du potentiel image classique ressenti par un électron traversant la jonction plano-sphérique.

Abstract - We present an exact method for the fast computation of a) the electrostatic potential distribution between two biased planar, parallel electrodes containing a spherical protrusion or an isolated conducting, spherical insertion and b) the classical image charge potential experienced by an electron erossing the plano-spherical junction.
\end{abstract}

\section{1 - INTRODUCTION}

Interest in tunneling through non planar MVM junctions has recently been enhanced by the advent of the Scanning Tunneling Microscope (STM) ${ }^{1}$.

Theoretical mode $1 \mathrm{~s}^{2}, 3,4$ have been constructed aiming at a better understanding of the observed spatial resolution of the microscope.

Prior to this, theoretical work ${ }^{5}$ had been concerned with nonplanar tunneling in metal whisker diodes which have a geometry similar to STM and which were instrumental in establishing the recently defined velocity of light ${ }^{6}$. Also, tunneling through curved interfaces occurs in electroluminescent MOM junctions ${ }^{7}$ where the luminescence is enhanced by roughness.

In discussing the tunneling in STM, it may be important to use realistic models of the junctions ${ }^{8}$. In one such model the tip surface is a planar conductor provided with a hemispherical protrusion which models an atomic cluster through which the tunnel current is believed to pass in the actual device. The actual imperfections are not expected to have such regular shapes but this model permits a theoretical analysis and is expected to give a first approximation to actual experimental conditions. The counter electrode representing the surface to be examined by STM is here a flat metal conductor separated from the tip by a vacuum gap.

In this paper we report on methods of solving two problems :

1) Determination of the three-dimensional potential barrier due to the external bias field. That is, given the potentials at the two electrodes, determine the poten-

\footnotetext{
+ This research was supported in part by the NATO Research Grants Program, Grant no 1902, Scientific Affairs Division, Brussels, Belgium and the Office of Naval Research, Arlington, Virginia, Contract No. N00014-82-K-0702.
} 
tial distribution in the space between them.

2) Determination of the classical image charge potential. That is, with the electrodes grounded and a point charge in the space between them, calculate the potential energy of the system as a function of the position of the point charge.

The sum of these two potential barriers represents the classical vacuum potential barrier across which the electrons in a STM tunnel.

The solution for the static potential obtained here could also be derived by solving Maxwe11's equations and boundary conditions for the corresponding quasi static problem in which the metal electrodes are represented by a local, frequency-dependent dielectric function $\varepsilon(\omega)$. Such an approach, which would also give the resonant modes of the MVM junction, has recently been adopted by Ruppin ${ }^{9}$ (who used a general method due to Berreman ${ }^{10}$ ) for a single planar electrode with one hemispherical bump, in the context of Surface Enhanced Raman Scattering.

As expected, for realistic values of the geometrical length parameters, the tunneling barrier exhibits strong deviations from the planar MVM barrier of the same materials. In particular, substantial narrowing of the barrier along the axial direction of the tip and large barrier asymmetry with respect to bias reversal are obtained. Graphical. representation of these numerical results will be presented and discussed.

\section{II - STATIC BIAS POTENTIAL DISTRIBUTION}

We will treat the general case of fig. 2 which covers all situations of partially protruding spheres $(a \leqslant R)$ or isolated spherical inclusions $(a>R)$ such as found in roughened electroluminescent MOM junctions.

The first thing we do is to reflect in the plane $z=0$ both the vacuum gap and the sphere of fig. 2, as shown in fig. 3. It is easy to convince oneself that the potential distribution in the upper half $(z>0)$ of the double gap coincides, by symmetry, with the potential of the actual junction of fig. 2 .

The potential at $\vec{r}$ would be known if we knew the induced surface charge density $\sigma$ on a17 surfaces. In fact, the knowledge of the surface charge density $\sigma\left(\vec{r}_{0}\right)$ on the upper sphere will suffice if we observe that

i) by symmetry, the surface charge density on the lower sphere is identical to $\sigma\left(\vec{r}_{0}\right)$ and

ii) employing the method of images to satisfy the boundary conditions, the surface charge density on the planar conductors at $d$ and $-d$ can be replaced by an infinite series of spherical charge distributions obtained by imaging the two spheres in the twin mirrors at $d$ and $-d$.

Thus the problem is entirely reduced to calculating the potential due to a linear array of identical spheres, clustered by pairs, and all carrying the same charge density $\sigma\left(\vec{r}_{0}\right)$ as the real sphere $S_{0}$. It is clear that if we obtain the potential at $\vec{r}$ due to $S_{0}$ alone, we can obtain the total potential by adding up the image sphere contributions through suitable translations of the $z$ coordinate. Let us write the axially symmetric total potential at $\vec{r}=(\rho, z)$ as

$V(\rho, z)=v_{0} \frac{z}{d}+V^{\prime}(\rho, z)$

where we have separated the simple planar gap contribution $v_{0} z / d$ which must be recovered for asymptotically large 0 . The perturbation $V^{\prime}$ satisfies the boundary cond $i-$ tions

$V^{\prime}=0$

for $z=0$

$v^{\prime}=0$

for $z= \pm d$

(a)

$V^{\prime}=-\frac{V_{0}}{d} z$ on $s_{0}$ 
Let us expand $\sigma$ in Legendre polynomials :

$\sigma\left(\vec{r}_{0}\right)=\sum_{\ell=1}^{\infty} A_{\ell} P_{\ell}\left(\cos \theta_{0}\right)$

where the $\ell=0$ term is excluded for charge neutrality. The potential due to the real sphere is given by

$V_{0}^{\prime}(\vec{r})=R^{2} \int_{S_{0}} d \Omega_{0} \frac{\sigma\left(\theta_{0}\right)}{\left|\vec{r}_{a}-\vec{r}_{0}\right|}$

where $\vec{r}$ is the position of point $P$ with respect to the center of the sphere $S_{0}$. Inserting the generating function of the $P^{\prime} s$

$\frac{1}{\left|\vec{r}_{a}-\vec{r}_{0}\right|}=\sum_{k=0}^{\infty} \frac{R^{k}}{r_{a}^{k+1}} P_{k}(\cos \alpha)$

and using the addition theorem of spherical harmonics

$P_{k}(\cos \alpha)=\frac{4 \pi}{2 k+1}-\sum_{m=-k}^{+k} \gamma_{k}^{m}{ }^{\star}\left(\theta_{0}, \phi_{0}\right) Y_{k}^{m}(\theta, \phi)$

in (4) gives

$V_{0}^{\prime}=4 \pi R^{2} \sum_{\ell k m} A_{\ell} \frac{R^{k}}{2 k+1} \frac{1}{r_{a}^{k+1}} Y_{k}^{m}(\theta, \phi) \int_{S_{0}} d \Omega_{0} P_{\ell}\left(\cos \theta_{0}\right) Y_{k}^{m_{k}^{\star}}\left(\theta_{0}, \phi_{0}\right)$.

Orthogonality of the $Y_{k}^{m_{1}} s$ reduces $V_{0}^{1}$ to

$V_{0}^{\prime}=\sum_{\ell=1}^{\infty} B_{\ell} \frac{1}{\left(\rho^{2}+z_{a}^{2}\right)^{\frac{\ell+1}{2}}} P_{\ell}\left(\frac{z_{a}}{\sqrt{\rho^{2}+z_{a}^{2}}}\right)$

where $z_{a}=z-a$, and $B_{\ell}$ are new unknown coefficients, proportional to $A_{\ell}$.

Now we can add contributions to the potential from all spheres centered at $z_{n}=$ $\pm(a+2 n d), n=1,2, \ldots$. The final result is

$$
\begin{aligned}
V^{\prime}(\vec{r})= & \sum_{\ell=1}^{\infty} B_{\ell} F_{\ell}(\rho, z) \\
F_{\ell}(\rho, z)= & \sum_{n=-\infty}^{\infty}\left[\dot{\rho}^{2}+(z-2 n d-a)^{2}\right] \quad P_{\ell}\left[\frac{z-2 n d-a}{\sqrt{\rho^{2}+(z-2 n d-a)^{2}}}\right] \\
& -(-1)^{\ell}\left[\rho^{2}+(z+2 n d+a)^{2}\right] \quad P_{\ell}\left[\frac{z+2 n d+a}{\sqrt{\rho^{2}+(z+2 n d+a)^{2}}}\right]
\end{aligned}
$$


It is easy to verify that $F_{l}$ is antisymmetric (change $n$ into $-n$ )

$F_{\ell}(\rho,-z)=-F_{\ell}(\rho, z)$

which guarantees the bouncary condition (3-a). By virtue of the image construction, $F_{\ell}$ also satisfies (the $n$th term cancels the $-(n+1)$ th term) $F_{\ell}(\rho,+d)=0$ which güarantees the boundary condition (3-b). Finally, the unknown coefficients $B_{\ell}$ are determined by imposing the last condition (3-c) :

$$
\begin{gathered}
\sum_{\ell=1}^{\infty} B_{\ell} F_{\ell}(\rho, z)=-\frac{V_{0}}{d} z \\
\text { for } \quad a-R \leqslant z \leqslant a+R, \\
\rho^{2}=R^{2}-z^{2}
\end{gathered}
$$

The $B_{\ell}$ coefficient for $\ell$ up to an upper multipolar limit $L$ can be found as the solutions of a set of $L$ linear equations obtained by imposing the condition (11) at $L$ distinct values of $z$ suitably chosen in the indicated interval. However it is more accurate to determine the first $L$ coefficients by least square adjusting ${ }^{1}$ them to a much larger number $N \gg L$ of equations (11) written for $N$ distinct values of $z$.

For a hemispherical protrusion $(a=0)$, the solution (8) (9) reduces to

$$
\begin{aligned}
& V^{\prime}(\vec{r})=\sum_{\ell=1}^{\infty} C_{\ell} F_{\ell}(\rho, z) \\
& F_{\ell}(\rho, z)=\sum_{n=-\infty}^{\infty}\left[\rho^{2}+(z+2 n d)^{2}\right]^{-\ell} P_{2 \ell-1}\left[\frac{z+2 n d}{\sqrt{\rho^{2}+(z+2 n d)^{2}}}\right]
\end{aligned}
$$

and, by symmetry, involves only odd multipoles. It turns out that the convergence of the $n$-summation over the images in (14) is very fast $(n \leqslant 10$ suffices to get the $F_{\&}$ with $10^{-5}$ accuracy). Regarding the multipole expansion, taking $\ell \leqslant 3$ proves sufficient to determine the $C_{l}{ }^{\prime} s$ with $10^{-5}$ accuracy. A few equipotentials are $i 1-$ lustrated in fig. 4. The potential values along the axial z direction $(\rho=0)$ are plotted in fig. 5. The characteristic buldging out of the potential, as compared to the linear behaviour of a planar MVM junction, is clearly seen. It reflects the enhancement of the electric field at the sphere apex.

\section{III - ELECTRON MULTIPLE IMAGE}

The construction of the self image potential of the tunneling electron is straightforward when using an appropriate sequence of multiple images. We illustrate the principle of the method for the case of a hemispherical protrusion which, as is we11 known, presents the simplifying feature of requiring only three images in the non planar electrode (fig. 6).

Let us denote $\left(q, \rho_{q}, z_{q}\right)$ the charge and position of the tunneling particle. This has exactly three images in the lower electrodes given by $\left(q^{\prime}, \rho_{q^{\prime}}, z_{q^{\prime}}\right),\left(-q^{\prime}, \rho_{q^{\prime}},-z_{q^{\prime}}\right)$ and $\left(-q, \rho_{q},-z_{q}\right)$ where

$q^{\prime}=-q \frac{R}{r_{q}} \quad$ and

$\frac{z_{q^{\prime}}}{z_{q^{\prime}}}=\frac{\rho_{q^{\prime}}}{\rho_{q}}=\frac{R^{2}}{r_{q}^{2}}$ 
The source charge $q$ and its three images in the lower electrode are considered as the first generation of an infinite set of generations constructed as shown in fig. 6 . The second generation, also having 4 charges, is simply the images in the plane $z=d$ of the previous charges. Each of the new charges in the upper electrode gives rise to exactly three new image charges in the lower electrode and so forth. Thus, the $2 n$th and $(2 n-1)$ th generations have the same number of charges, namely $4.3^{n-1}$. $(n=1,2, \ldots, \infty)$.

Once the image charge array $q_{j}$ has been constructed, the self image potential energy of the source charge $q$ is written as

$w=+\frac{1}{2} q \sum_{i} \frac{q_{j}}{\left|\vec{r}-\vec{r}_{j}\right|}$

where the factor $1 / 2$ takes account of the induced nature of the images ${ }^{12}$.

Each charge generation being neutral, the potential calculated from (16) converges very fast : 10 generations suffice to get $W$ with an accuracy of $10^{-5}$. One typical result is illustrated in fig. 7 for $\rho=0$. The image potential energy is also slightly asymmetrical as expected and, of course, diverges at $z=R$ and $z=d$. If desired, this can be easily corrected in the usual way by withdrawing the image planes slightly inside the conductors. These image potential values must be added to the static bias potential of fig. 4 to construct the tunnel barrier through the protrusion ${ }^{12}$.

\section{IV - CONCLUSION}

The most important result of the present calculations is to demonstrate the dominant role of the classical multiple image potential in determining the shape of the vacuum barrier in the STM junction where the static bias potentials are on the order of $10 \mathrm{meV}$. Consequently, it is essential to recognize the nonseparability of this barrier which precludes the use of a one-dimensional transmission coefficient for tunneling calculations in a realistic model of the STM. A more complete discussion of the calculated barriers and the significance in three-dimensional tunneling will be published elsewhere.

\section{REFERENCES}

1. G. Binnig, H. Bohrer, Ch. Berber and E. Weibe1, Phys, Rev. Letters 49, 57 (1982); Phys. Rev. Letters 50, 120 (1983).

2. J. Tersoff and D. Hamann, Phys. Rev. Lett. 50, 1998 (1983).

3. N. Garcia, C. Ocal and F. Flores, Phys. Rev. Lett. 50, 2002 (1983).

4. A. Baratoff, Europhysics Conference Abst. 7b, 364 (1983).

5. T.E. Feuchtwang, P.H. Cut]er, N.M. Miskowsky and A.A. Lucas, "Quantum Metrology and Fundamenta? Physical Constants", ed. by P.H. Cutler and A.A. Lucas, NATO ASI Series B, vol. 98, p. 529 (1983). Plenum Press, New York.

6. K.M. Evenson, "Quantum Metrology and Fundamental Physical Constants", ed. by P.H. Cutler, N.M. Miskowsky and A.A. Lucas, NATO ASI Series B, vol. 98, p. (1983). Plenum Press, New York.

7. A. Adamson, J.C. Wyss and P.K. Hansma, Phys. Rev. Lett. 32, 545 (1978).

8. T.E. Feuchtwang, P.H. Cutler and N.M. Miskowsky, Phys. Lett. 99A, 167 (1983).

9. R. Ruppin, Solid State Comm. 39, 903 (1981).

10. D:W. Berreman, Phys. Rev. 163, 855 (1967); Phys. Rev. B1, 381 (1970).

11. G.E. Forsythe, M.A. Malcolm and C.B. Moler, Computer Methods for Mathematical Computations. Prentice-Hal1, Englewood Cliffs, 1977.

12. Proper inclusion of the factor $1 / 2$ in the image potential is important for calculating the tunnel current, as shown in N.M. Miskowsky, P.H. Cutler, T.E. Feuchtwang and A.A. Lucas, App 1. Phys. A27, 139 (1982). 


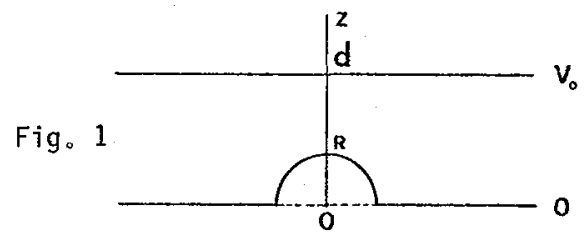

\section{Figure 1}

Model planar MVM junction with hemispherical protrusion.

\section{Figure 2}

Model planar MVM junction with spherical inclusion.

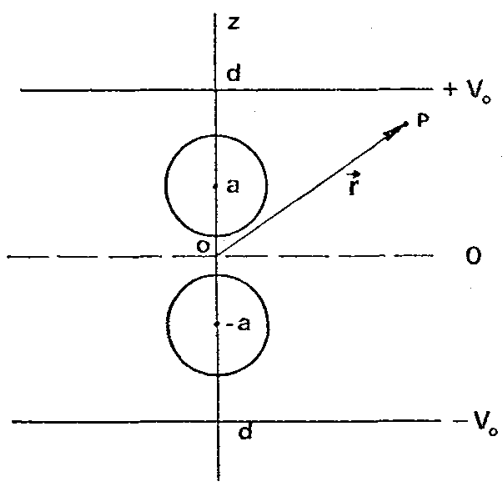

Figure 3

Antisymmetrical duplication of the junction of Fig. 2 


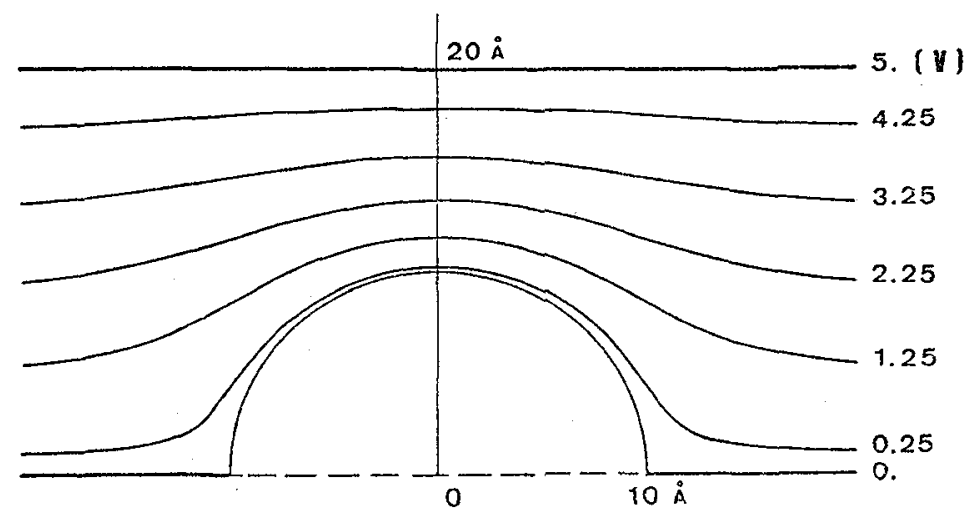

Figure 4

Equipotential Tines in the MVM junction biased to $5 \mathrm{~V}$.

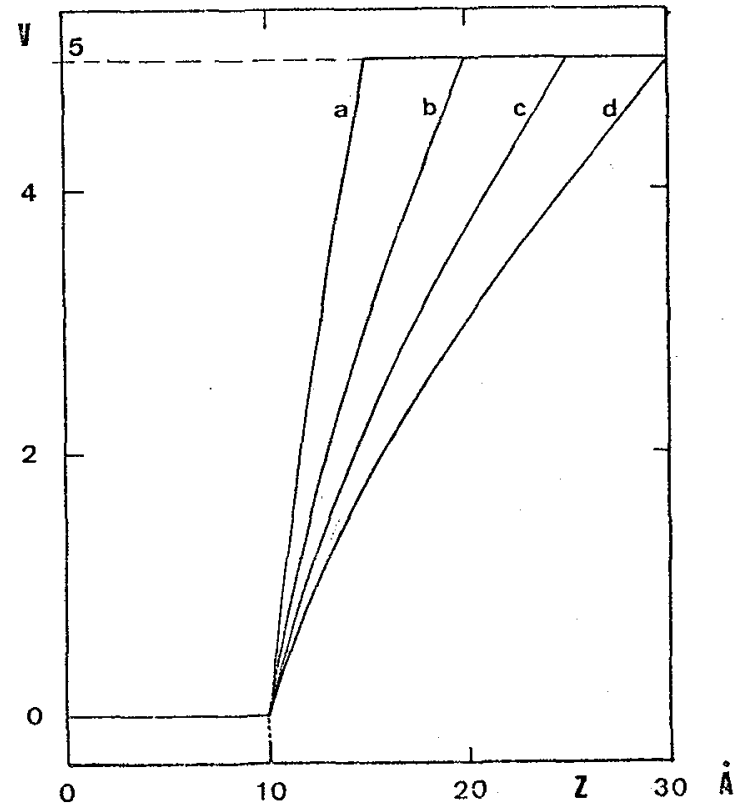

Figure 5

Potential distributions in the vacuum gap of a junction biased to $5 \mathrm{~V}$. $\mathrm{a}, \mathrm{b}, \mathrm{c}$ and $\mathrm{d}$ correspond to a width of $5,10,15$ and $20 \AA$ respectively. The hemispherical protrusion has a radius of $10 \AA$. 


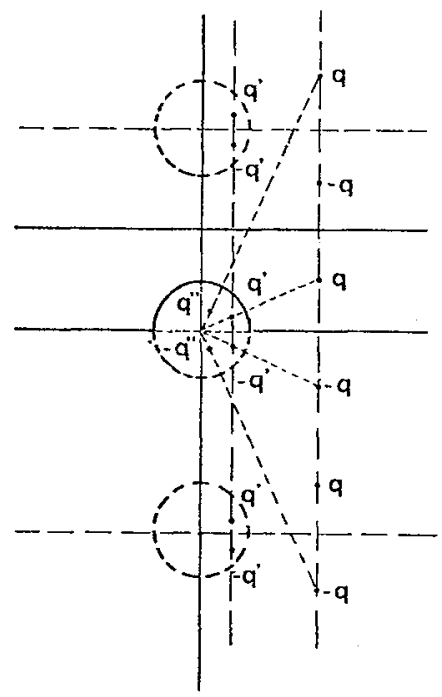

Figure 6 - Iteration method to generate the set of multiple images of the object charge $q$ in the vacuum gap of a junction with hemispherical protrusion.

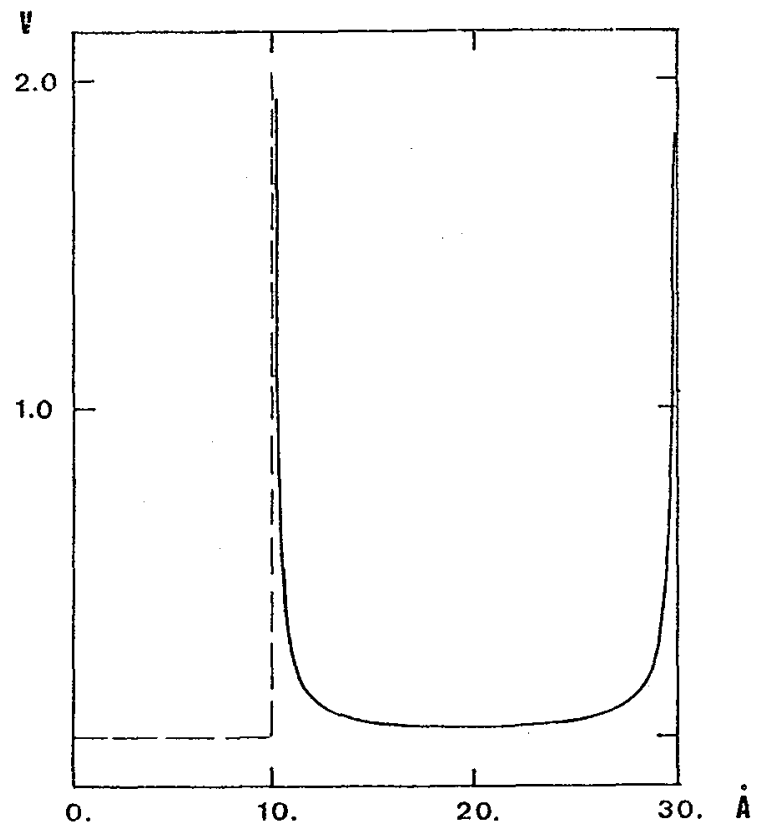

Figure 7 - Self-image potential along the symmetry axis for a charge in a junction of $30 \AA$ width having a hemispherical protrusion of $10 \AA$. 